\title{
Non-alcoholic fatty liver disease (NAFLD): C.U.R.I.A.Mo. lifestyle intervention and hepatic steatosis indexes
}

\author{
Created by: Roberto Pippi \\ Version received: 22 May 2020
}

\begin{abstract}
Non-alcoholic fatty liver disease (NAFLD) has an estimated prevalence of $20-30 \%$ in the general population and even higher in individuals with metabolic risk factors. The aim of this study was to evaluate the effect of a lifestyle intervention program on surrogate markers of hepatic steatosis in obesity and/or type 2 diabetes patients, enrolled in the C.U.R.I.A.Mo.(Centro Universitario di Ricerca Interdipartimentale Attività Motoria) trial. 102 subjects with type 2 diabetes, obesity or a BMI of at least $25 \mathrm{~kg} / \mathrm{m}^{2}$ with comorbidities, participated in the intensive phase of a multidisciplinary lifestyle intervention program at the Healthy Lifestyle Institute of the University of Perugia (C.U.R.I.A.Mo.).Six indices related to NAFLD (Visceral Adiposity Index, Fatty Liver index, Non-Alcoholic Fatty LiverDisease liver fat score and liver fat equation, hepatic steatosis index and TyG index) were calculated before and after a three-month multidisciplinary lifestyle intervention. The intervention improved the anthropometric and clinical parameters in the total population, the obese and/or diabetics. Data showed a significant weight loss, a reduced waist circumference, triglycerides, and an improvement in Mediterranean diet adherence. Hepatic steatosis indices were significantly reduced in the total population and in different subgroups (males, females, obesity and diabetes).
\end{abstract}

Non-alcoholic fatty liver disease (NAFLD) is a significant public health problem, with an estimated prevalence of 20$30 \%$ in the general population and even higher in individuals with metabolic risk factors (visceral obesity, type 2 diabetes, dyslipidemia, and/or insulin resistance) [1].

Patients with NAFLD show higher all-cause mortality and increased risk for liver-related death and cardiovascular disease [2][3]. Due to the pathophysiological link existing between visceral adiposity, insulin resistance, and hepatic steatosis, therapeutic interventions aimed at improving lifestyle are essential and are recommended at any stage of disease [4]. Dietary changes are recommended by Clinical Practice Guidelines shared by the three major European Associations most consistently involved in the care of NAFLD/NASH patients recommend in their shared Clinical Pratice Guidelines weight loss of $7 \%$, physical activity, reduced sedentary behavior, and dietary changes, all of which should be maintained throughout life [4].

The understanding of the overall prevalence of NAFLD is largely dependent on the criteria used for assessment. Liver biopsy represents the gold standard for diagnosis and the assessment of NAFLD severity. Although it can differentiate simple steatosis, steatohepatitis, and fibrosis, liver biopsy has certain drawbacks, as it is invasive, not feasible in all NAFLD patients, costly, suffers from sampling errors, has intra- and inter-observer variability, and must be limited to patients with increased risks of advanced fibrosis [5]. The mass screening of asymptomatic individuals using imaging modalities, such as ultrasonography and computed tomography, is not cost-effective because these studies are expensive. Hepatic ultrasound is highly operator-dependent and has limited repeatability and reproducibility [6] owing to subjective qualitative features of liver echogenicity. More precise imaging methodologies have been developed, including computed tomography (CT), magnetic resonance imaging (MRI), and spectroscopy (MRS); however, these are not widely available. CT provides objective assessment of hepatic X-ray attenuation, which is related to liver fat content. However, several factors other than fat (e.g., the presence of iron, copper, glycogen, fibrosis, or edema) confound attenuation values [7]. MRI and MRS have been validated and have been shown to be accurate for detection and quantification of hepatic steatosis. MR-based methods, however, are not used widely for screening because of cost, lack of availability, and lack of validated cutoffs to determine NAFLD. It is therefore appropriate to specify that the technique used to define normal liver fat influences the normal value. As a solution to this problem, in the past few years simple steatosis indexes were developed. These surrogate measurements are based on anthropometric parameters such as body mass index (BMI) and waist circumference (WC) and laboratory tests such as raised liver enzymes (aminotransferases and gamma-glutamyl transpeptidases-ALT, AST, and GGT), altered lipid levels (triglycerides and HDL-cholesterol-TG and C-HDL) and fasting glucose. Overall, steatosis biomarkers can accurately 
differentiate between the absence and the presence of steatosis [8].

The aim of this study is to evaluate the effect of a lifestyle intervention program on surrogate markers of hepatic steatosis in obese and/or type 2 diabetes patients, enrolled in the C.U.R.I.A.Mo. trial.

The lifestyle intervention improved the anthropometric and clinical parameters in the total population, in the obese and/or diabetics. Data collected at baseline and after three months showed significant weight loss, reduced waist circumference, triglycerides reduction and an improvement in eating habits with a change toward higher adherence to the Mediterranean diet. These data demonstrate the clinical efficacy of the C.U.R.I.A.Mo. intervention, a multidisciplinary and combined approach, for the improvement of lifestyle leading to an improvement in health.

Hepatic steatosis indices calculated before and after lifestyle intervention, showed a significant reduction in the total population and in different subgroups (males, females, obesity and diabetes) except for three indices: VAI for the obesity, males and females subgroups, HSI for the diabetes and TyG for obesity.

Recently it was observed that NAFLD patients with metabolic risk factors (among which is the presence of type 2 diabetes) require a larger weight loss $(\geq 10 \%)$ to produce the same beneficial effect on histological features of nonalcoholic steatohepatitis, in comparison to subjects with favorable risk factors (eg, absence of diabetes, BMI < 35) [9]. Nonetheless, this amount of weight loss may be achieved, and C.U.R.I.A.Mo. multidisciplinary intervention previously demonstrated its effective impact on body weight ${ }^{10]}$. This short-term analysis on steatosis indexes suggests that fat removal occurs rapidly in the course of lifestyle intervention, as also demonstrated by imaging techniques in other settings, both after restrictive diet ${ }^{[11]}$ and after exercise. The correlation analysis suggests some links between steatosis index improvement and waist circumference and triglycerides reductions. This is in line with the scientific literature where a direct association between remission of NAFLD (proven by proton magnetic resonance spectroscopy - and body weight and waist circumference reduction has been consistently demonstrated. All these parameters are in keeping with loss of visceral fat, that has a key role in the pathogenesis of hepatic steatosis.

One of the major limitations of this retrospective study is that the hepatic indexes used only roughly represent the degree of inflammation and lipid infiltration of the liver while the gold standard test for this purpose is histological examination [,. Furthermore, indexes were calculated retrospectively. For this reason, it was not possible to evaluate all the indices in the entire population referred to C.U.R.I.A.Mo., due to the lack of the clinical data necessary for the calculation.

Due to uncertainties surrounding treatment options, screening for hepatic steatosis is not currently performed in the general population and high-risk groups attending diabetes or obesity clinics, such as those who participated in the clinical trial of C.U.R.I.A.Mo., but current Guidelines from European [4] and National association stress the importance of such assessment. Despite the limitations related to their poor specificity, regarding which further studies are needed, the indices of hepatic steatosis are cheap, reproducible, and can be calculated for many people who have access to a basic health plan. Unfortunately, these recommendations were not operative at the time of treatment initiation, and only limited information could be retrospectively derived. In the context of an intensive lifestyle intervention, directed at high metabolic risk populations, hepatic steatosis indexes may be useful in identifying individuals who, after the lifestyle program, still have elevated hepatic indices and must undergo imaging assessment and specific hepato-protective therapy.

To the best of our knowledge this is the only study that has evaluated the performance of six hepatic indices in subjects with high metabolic risk that undergo a multidisciplinary and intensive lifestyle intervention.

Our findings support current recommendations for the management of hepatic steatosis, in which weight loss, obtained through lifestyle modification, represents the first line therapy, even in diabetes subjects.

In the diabetic population more, and more extensive, investigations on the effect of lifestyle improvement on hepatic steatosis are needed, because people with diabetes are at greater risk of progression of liver disease, developing more severe forms of NAFLD such as steatohepatitis on alcoholic and hepatocellular carcinoma [12][13]. The clinical protocol of C.U.R.I.A.Mo, which consists of the shared and joint action of various professionals, represents a care 
model aimed at achieving the psychophysical health of the whole person. The growth of awareness in the population at risk and actually maintaining a healthy lifestyle are effective tools in the fight against NAFLD.

\section{References}

1. Williams, C.D.; Stengel, J.; Asike, M.I.; Torres, D.M.; Shaw, J.; Contreras, M.; Landt, C.L.; Harrison, S.A.Prevalence of nonalcoholic fatty liver disease and nonalcoholic steatohepatitis among a largely middle-agedpopulation utilizing ultrasound and liver biopsy: A prospective study.Gastroenterology2011,140, 124-131.

2. Targher, G.; Day, C.P.; Bonora, E. Risk of cardiovascular disease in patients with nonalcoholic fatty liverdisease.N. Engl. J. Med.2010,363, 1341-1350.

3. Arslan, U.; Türko glu, S.; Balciǒ glu, S.; Tavil, Y.; Karakan, T.; Cengel, A. Association between nonalcoholicfatty liver disease and coronary artery disease.Coron. Artery Dis.2007,18, 433-436

4. EASL-EASD-EASO. Clinical Practice Guidelines for the management of non-alcoholic fatty liver disease.J. Hepatol.2016,64, 13881402.

5. Chalasani, N.; Younossi, Z.; Lavine, J.E.; Diehl, A.M.; Brunt, E.M.; Cusi, K.; Charlton, M.; Sanyal, A.J.The Diagnosis and Management of Non-Alcoholic Fatty Liver Disease: Practice Guideline by the AmericanAssociation for the Study of Liver Diseases, American College of Gastroenterology, and the AmericanGastroenterological Association.Hepatology2012,55, $2005-2023$.

6. Strauss, S.; Gavish, E.; Gottlieb, P.; Katsnelson, L. Interobserver and intraobserver variability in thesonographic assessment of fatty liver.AJR Am. J. Roentgenol.2007,189, 320-323.

7. Reeder, S.B.; Cruite, I.; Hamilton, G.; Sirlin, C.B. Quantitative assessment of liver fat with magnetic resonanceimaging and spectroscopy.J. Magn. Reson. Imaging2011,34, 729-749.

8. Fedchuk, L.; Nascimbeni, F.; Pais, R.; Charlotte, F.; Housset, C.; Ratziu, V. Performance and limitation ofsteatosis biomarkers in patient with nonalcoholic fatty liver disease.Aliment. Pharmacol. Ther.2014,40,1209-1222.

9. Vilar-Gomez, E.; Martinez-Perez, Y.; Calzadilla-Bertot, L.; Torres-Gonzalez, A.; Gra-Oramas, B.;Gonzalez-Fabian, L.; Friedman, S.L.; Diago, M.; Romero-Gomez, M. Weight loss through lifestyle modificationsignificantly reduces features of nonalcoholic steatohepatitis.Gastroenterology2015,149, 367-378.

10. SbromaTomaro, E.; Pippi, R.; Reginato, E.; Aiello, C.; Buratta, L.; Mazzeschi, C.; Perrone, C.; Ranucci, C.; Tirimagni, A.; Russo, A.; et al. Intensive lifestyle intervention is particularly advantageous in poorlycontrolled type 2 diabetes.Nutr. Metab. Cardiovasc. Dis.2017,27, 688-694.

11. Browning, J.D.; Davis, J.; Saboorian, M.H.; Burgess, S.C. A Low-Carbohydrate Diet Rapidly and DramaticallyReduces Intrahepatic Triglyceride Content. Hepatology2006,44, 487-488.

12. Bugianesi, E.; Leone, N.; Vanni, E.; Marchesini, G.; Brunello, G.; Carucci, P.; Musso, A.; De Paolis, P.;Capussotti, L.; Salizzoni, M.; et al. Expanding the natural history of nonalcoholic cirrhosis to hepatocellularcarcinoma.Gastroenterology2002,123, 134-140.

13. Porepa, L.; Ray, J.G.; Sanchez-Romeu, P.; Booth, G.L. Newly diagnosed diabetes mellitus as a risk factor forserious liver disease.CMAJ2010,182, 526-531.

\section{Keywords}

Non-alcoholic fatty liver disease; obesity; type 2 diabetes mellitus; lifestyle; exercise 